\title{
Hydralazine prn, should we really use it?
}

\author{
Alejandro Velasco MD, Michael Phy DO
}

Physicians commonly approach hospitalized patients with severe asymptomatic hypertension by treating a number rather than the patient as a whole. However, the use of intravenous hydralazine to acutely lower blood pressure (BP) in this setting can have its own risks. Besides having an unpredictable pharmacokinetic profile, it can be harmful in conditions such as myocardial infarction and aortic dissection. ${ }^{1}$ Campbell, et al. performed an observational study describing the appropriateness, efficacy, and side effects of intravenous hydralazine doses given in hospitalized patients. ${ }^{2}$ In this study only $2 \%$ of patients had evidence of hypertensive emergency symptoms justifying use of an IV antihypertensive medication. Physicians evaluated only $7.5 \%$ of patients prior to hydralazine dosing, and just $25 \%$ of them had an adjustment in their long-term BP medications. Furthermore, a significant proportion of patients (16\%) experienced side effects, such as hypotension, dizziness, and lightheadedness. As an assessment of routine practices in the in-patient management of BP elevations, Weder, et al. performed a retrospective review of patients receiving prn doses of hydralazine and/or labetalol who were not admitted specifically for management of hypertension. Of the 2189 patients analyzed in this study, only $2.9 \%$ had a diagnosis for which rapid BP reduction with intravenous agents would be indicated. Hydralazine was commonly prescribed to be given every hour, which is not in agreement with the expert recommendation of $10-20 \mathrm{mg}$ every 4 to 6 hours. Notably, the mean length of stay was significantly increased in patients who received prn IV antihypertensive (12.0 \pm 15.9 days) compared to patients in whom medication was ordered but not given

Corresponding author: Alejandro Velasco, MD Contact Information: alejovela@hotmail.com DOI: 10.12746/swrccc2015.0310.131
$(7.1 \pm 10.4$ days, $p<0.001) \cdot{ }^{3}$ It should be noted that subjects receiving prn medications were significantly older $(60.5 \pm 16.5$ vs. $54.4 \pm 18.8$ years, $p<0.001)$, and this could have affected the results.

Unfortunately, there are no guidelines or standard recommendations to guide the management of acute elevations of blood pressure in the hospital setting. Although hydralazine has proven useful in cases of pre-eclampsia, it is reasonable to prefer a slow titration of BP medications rather than frequent IV hydralazine doses in the absence of target organ compromise during hypertensive episodes in hospitalized patients.

Author Affiliation: Alejandro Velasco is a Hypertension Fellow in the cardiology division at The University of Texas Southwestern Medical Center in Dallas. Michael Phy is a general internist in the Department of Internal Medicine at Texas Tech University Health Sciences Center in Lubbock.

Received: 02/06/2015

Accepted: 04/09/2015

Reviewers: Vaqar Ahmed MD

Published electronically: 4/15/2015

Conflict of Interest Disclosures: None

\section{REFERENCES}

1. Ludden TM, Shepherd AM, McNay JL, Jr., Lin MS. Effect of intravenous dose on hydralazine kinetics after administration. Clinical pharmacology and therapeutics 1983;34:14852 .

2. Campbell P, Baker WL, Bendel SD, White WB. Intravenous hydralazine for blood pressure management in the hospitalized patient: its use is often unjustified. Journal of the American Society of Hypertension : JASH 2011;5:473-7. 3. Weder AB, Erickson S. Treatment of hypertension in the inpatient setting: use of intravenous labetalol and hydralazine. Journal of clinical hypertension 2010;12:29-33. 